Statistica Neerlandica (2009) Vol. 63, nr. 3, pp. 334-346

doi:10.1111/j.1467-9574.2009.00426.x

\title{
Expert opinion versus expertise in forecasting
}

\author{
Philip Hans Franses* and Michael McAleer** \\ Econometric Institute, Erasmus University Rotterdam, PO Box 1738, \\ NL-3000 DR Rotterdam, The Netherlands \\ Rianne Legerstee $\dagger$ \\ Econometric Institute and Tinbergen Institute, Erasmus University \\ Rotterdam, PO Box 1738, NL-3000 DR Rotterdam, The Netherlands
}

Expert opinion is an opinion given by an expert, and it can have significant value in forecasting key policy variables in economics and finance. Expert forecasts can either be expert opinions, or forecasts based on an econometric model. An expert forecast that is based on an econometric model is replicable, and can be defined as a replicable expert forecast (REF), whereas an expert opinion that is not based on an econometric model can be defined as a non-replicable expert forecast (Non-REF). Both REF and Non-REF may be made available by an expert regarding a policy variable of interest. In this paper, we develop a model to generate REF, and compare REF with Non-REF. A method is presented to compare REF and Non-REF using efficient estimation methods, and a direct test of expertise on expert opinion is given. The latter serves the purpose of investigating whether expert adjustment improves the model-based forecasts. Illustrations for forecasting pharmaceutical stock keeping unit (SKUs), where the econometric model is of (variations of) the autoregressive integrated moving average model (ARIMA) type, show the relevance of the new methodology proposed in the paper. In particular, experts possess significant expertise, and expert forecasts are significant in explaining actual sales.

Keywords and Phrases: direct test, efficient estimation, expert opinion, replicable expert forecasts, generated regressors, non-replicable expert forecasts.

There are as many opinions as there are experts.

Franklin D. Roosevelt, 32nd US President (1933-1945)

\section{Introduction}

Econometric models are useful for forecasting key policy variables in economics and business. Sometimes the outcomes of these models are adjusted by experts, and there

\footnotetext{
*franses@ese.eur.nl

**michael.mcaleer@gmail.com

†legerstee@ese.eur.nl 
are many reasons why an expert could do so (see, e.g. Goodwin, 2000, for a useful summary). Expert adjustments to model-based forecasts occur in economics (see, e.g., Franses, Kranendonk and Lanser, 2007; Romer and Romer, 2008), and in business (see Bunn and Salo, 1996; Franses and Legerstee, 2009, for an extensive empirical survey). Interestingly, the inclination of experts to adjust model-based forecasts is independent of the size of the econometric model (see Franses, 2008). Indeed, forecasts from both large-scale macro-econometric models and from smallscale ARIMA models might be adjusted by an expert.

In this paper, we examine to what extent we can capture expert adjustment in an econometric modeling framework, with the ultimate purpose of investigating whether expert adjustment improves the model-based forecasts. We assume that there is data available on the expert forecast, model forecast, and of course on the realizations. Preferable, we also have access to variables that could be relevant to predict these realizations. This means that our methodology can be applied to any kind for expert forecasts, also those of Greenbook and of the Survey of Professional Forecasters.

For this purpose, we need some definitions in order to be perfectly clear where we are heading. As is well known, a forecast is an inference about an event that was not observed at the time of the inference. Forecasts generated from econometric models are replicable, and this feature will become transparent next.

Expert opinions are given by experts, and much has been made of the value of expert opinions, especially with regard to their potential value in forecasting key policy variables in economics and finance. However, expert forecasts that are replicable (REF) need to be distinguished from expert opinions that are not. REF are forecasts made by an expert, or by others using the same information that is available to the expert, using an appropriate econometric model. In contrast, expert opinions are non-replicable forecasts (Non-REF) provided by experts relating to a policy variable of interest. Although expert opinions may be expressed as quantitative measures, they inherently contain a qualitative (or latent) component, namely expertise, and hence also contain measurement error.

The preceding discussion leads to the following three definitions.

Definition 1. Expertise is latent.

DEFINITION 2. Expert forecasts from an econometric model are REF.

\section{Definition 3. Expert opinions are Non-REF.}

Although expertise is unobserved, it can be estimated using an appropriate econometric model. The primary purpose of this paper is to develop an econometric model to generate REF, and to compare REF with Non-REF. A method is presented to () 2009 The Authors. Journal compilation (c) 2009 VVS. 
compare REF and Non-REF using efficient estimation methods, and a direct test of expert opinion is given.

The plan of the remainder of the paper is as follows. Section 2 presents the econometric model specification, compares REF and Non-REF, considers optimal forecasts and efficient estimation methods, and presents a direct test of expertise on expert opinion. Some relevant empirical examples are presented in section 3. Concluding comments are given in section 4 .

\section{Model specification}

In this section, we develop an econometric model to generate REF, and to enable a comparison to be made with Non-REF.

\subsection{Econometric model}

Let the econometric model be given as:

$$
y=X_{1} \beta_{1}+u_{1}, \quad u_{1} \sim\left(0, \sigma_{1}^{2} I\right),
$$

where $y$ is a $(T \times 1)$ vector of the dependent variable, $X_{1}$ is a $\left(T \times k_{1}\right)$ matrix of explanatory variables, where the first column corresponds to the intercept term, and $u_{1}$ is a $(T \times 1)$ vector of errors. The $y$ vector and $X_{1}$ matrix are observed, and $X_{1} \subset$ $I_{-1}^{M}$, where $I_{-1}^{M}$ is the information set of the econometric modeler at time $t-1(t=$ $2, . ., T)$.

It is assumed that the econometric model is appropriately specified, that is, it passes relevant diagnostic checks, that $I_{-1}^{M}$ contains publicly known information, and $E\left(X_{1}^{\prime} u_{1}\right)=0$. Under these conditions, oridinary least squares (OLS) in equation (1) is consistent and efficient, and hence is optimal in estimation. Moreover,

$$
\hat{y}_{1}=X_{1} \hat{\beta}_{1}=X_{1}\left(X_{1}^{\prime} X_{1}\right)^{-1} X_{1}^{\prime} y=P_{1} y,
$$

where $P_{1}=X_{1}\left(X_{1}^{\prime} X_{1}\right)^{-1} X_{1}^{\prime}$ is the standard 'hat' matrix.

If the model is correctly specified, under the assumption of mean squared error (MSE) loss, the optimal forecast of $y$, given the information set $I_{-1}^{M}$, is its conditional expectation (see Patton and Timmermann, 2007a,b).

\subsection{Replicable and non-replicable expert forecasts}

The fitted values (or in-sample model-based 'forecasts') of $y$ from equation (2) are made available to an expert, who is expected to improve on the forecast of $y$ through adding information to $\hat{y}_{1}$. The expertise possessed by the expert is latent as it is not publicly available, and may not even be quantifiable to the expert. Expertise is, in effect, a trade secret, which may be known only to the expert. A trade secret is defined under the Uniform Trade Secrets Act of 1985 as 'information that derives independent economic value, actual or potential, from not being generally (c) 2009 The Authors. Journal compilation (c) 2009 VVS. 
known, and not being readily ascertainable by proper means, by any other person, and is the subject of efforts that are reasonable under the circumstances to maintain its secrecy' (see Hoti, Mcaleer and Slottje, 2006, for further details). If expertise can be estimated through an appropriate econometric model, the public would be able to replicate expertise if they were to have access to the expert's information set.

Therefore, an important issue to be addressed is whether an expert forecast can be replicated. Let a $(T \times 1)$ vector $X_{2}$ represent observable expert opinion, as announced by an expert. The connection between the observed expert opinion and latent expertise is given as:

$$
X_{2}=X_{2}^{*}+\eta, \quad \eta \sim\left(0, \sigma_{\eta}^{2} I\right),
$$

where $X_{2}, X_{2}^{*}$, and $\eta$ are $(T \times 1)$ vectors, $X_{2}$ denotes expert opinion, $X_{2}^{*}$ represents latent expertise, $\eta$ is the measurement error, and $X_{2}^{*}$ and $\eta$ are assumed to be uncorrelated.

Let the observed expert opinion be given as:

$$
X_{2}=W \delta+\eta, \quad \eta \sim\left(0, \sigma_{\eta}^{2} I\right),
$$

where the $\left(T \times k_{2}\right)$ matrix $W$ is in the information set available to the expert at time $t-1$, and the first column of $W$ is the unit vector. It is assumed that $E\left(W^{\prime} \eta\right)=0, \delta$ is a $\left(k_{2} \times 1\right)$ vector of constant parameters, and that

$$
W=\left\{\hat{y}_{1}, W_{1}\right\} \subset I_{-1}^{E},
$$

which is the information set of the expert at time $t-1, W_{1}$ is $\left(T \times\left(k_{2}-2\right)\right)$, and $\hat{y}_{1}$ is available to the expert in providing an expert opinion, $X_{2}$. We assume that $W_{1}$ is also the information set of the analyst who studies the properties of expert forecast, but that this analyst does not necessarily need to know the model forecast.

Although the econometric model in equation (1) may be well specified, the expert may believe that an expert model is superior as it incorporates expertise. Hence, if the model in equation (4) is correctly specified, under the assumption of MSE loss, the optimal replicable expert forecast of $y$, given the information set $I_{-1}^{E}$, is its conditional expectation, so that the expert forecast is still optimal. OLS is consistent and efficient, and hence is optimal in estimation.

However, if the expert does not have an appropriate econometric model in forming expert opinion, the resulting Non-REF will not be optimal assuming a MSE loss function.

It follows from equation (4) and $I_{-1}^{E}$ that

$$
\mathrm{E}\left(X_{2} \mid I_{-1}^{E}\right) \equiv X_{2}^{*}=W \delta
$$

so that $W$ also denotes expertise as $X_{2}^{*}$ is a linear combination of the columns of $W$. The rational expectation estimate of $E\left(X_{2} \mid I_{-1}^{E}\right)$, which is an REF, is given as:

$$
\hat{X}_{2}^{*}=\hat{X}_{2}=W \hat{\delta}=W\left(W^{\prime} W\right)^{-1} W^{\prime} X_{2}=P_{W} X_{2},
$$

so that the estimate of the latent expertise, $X_{2}^{*}$, is equivalent to the estimate of the observable expert opinion, $X_{2}$. 
Remark 1 . The information set of the expert, $W$, includes $\hat{y}_{1}$ but does not necessarily include $X_{1}$.

REMARK 2. An REF can be consistently estimated as:

$$
\hat{X}_{2}^{*}=P_{W} X_{2}=\hat{X}_{2} \text {. }
$$

Remark 3. Expertise differs from expert opinion as $X_{2}-X_{2}^{*}=\eta$, and the difference can be estimated as $X_{2}-\hat{X}_{2}^{*}=X_{2}-\hat{X}_{2}$, namely the difference between Non-REF and REF, or the sample measurement error.

The expert's econometric model for forecasting $y$ is given by

$$
y=\delta_{0} \hat{y}_{1}+\beta_{2} X_{2}^{*}+u_{2}, \quad u_{2} \sim\left(0, \sigma_{2}^{2} I\right),
$$

where $\beta_{2}$ is a scalar parameter. As $X_{2}^{*}$ is latent and hence unobservable, an observable, and thereby estimable, version of equation (7) is given as:

$$
y=\delta_{0} \hat{y}_{1}+\beta_{2} \hat{X}_{2}+\varepsilon,
$$

where

$$
\begin{aligned}
\varepsilon & =u_{2}+\beta_{2}\left(X_{2}^{*}-\hat{X}_{2}\right) \\
& =u_{2}+\beta_{2}\left(W \delta-P_{W} X_{2}\right) \\
& =u_{2}+\beta_{2}\left(W \delta-P_{W}(W \delta+\eta)\right) \\
& =u_{2}-\beta_{2} P_{W} \eta
\end{aligned}
$$

REMARK 4. Under the null hypothesis that $\beta_{2}=0$ in equation (7), it follows that $\varepsilon=u_{2}$ in equation (9).

REMARK 5. Although $\hat{y}_{1}$ is not correlated with $\varepsilon$ in equation (9), the correlation between $\hat{X}_{2}$ and $\varepsilon$ is given by $-\beta_{2} \sigma_{\eta}^{2}\left(T-k_{2}\right)$. However, OLS estimation of the parameters in equation (8) is consistent as $\hat{X}_{2}$ is asymptotically uncorrelated with $\varepsilon$.

Remark 6. The null hypothesis $\delta_{0}=0$ in equation (8) is a test of whether the expert should use the model forecasts, $\hat{y}_{1}$, as a complement to REF, as given in $\hat{X}_{2}$.

Definition 4. An expert's forecast of y from equation (8) is given by

$$
\hat{y}_{E}=\hat{\delta}_{0} \hat{y}_{1}+\hat{\beta}_{2} \hat{X}_{2} \text {. }
$$

Under a MSE loss function, the forecast given in equation (10) is optimally relative to the expert's information set, $I_{-1}^{E}$. 
If $u_{1}$ in equation (1), $u_{2}$ in equation (7), and $\eta$ in equation (3) are mutually and serially uncorrelated, then

$$
E\left(\varepsilon \varepsilon^{\prime}\right)=E\left(u_{2} u_{2}^{\prime}\right)+E\left(P_{W} \eta \eta^{\prime} P_{W}\right) \beta_{2}^{2},
$$

and hence

$$
\Sigma_{\varepsilon}=\sigma_{2}^{2} I+\beta_{2}^{2} \sigma_{\eta}^{2} P_{W} .
$$

REMARK 7. Serial correlation and heteroskedasticity are generated in equation (11) through the measurement error, $\eta$, in $X_{2}$ in equation (3).

Remark 8. If the null hypothesis in equation (8) is $\beta_{2}=0$, then $\Sigma_{\varepsilon}=\sigma_{2}^{2} I$ in equation (11).

REMARK 9. Equations (7) and (8) can be interpreted as comprehensive approaches to testing non-nested hypotheses, namely the model-based forecast, $\hat{y}_{1}$, versus expertise, as captured in the latent variable, $X_{2}^{*}$, and observable variable, $\hat{X}_{2}$, respectively (for further details see, e.g. MCALEer, 1995).

\subsection{Efficient estimation}

In order to derive the conditions under which OLS estimation of the parameters in equation (8) is efficient, we appeal to Kruskal's theorem, which is necessary and sufficient for OLS to be efficient (see Fiebig, MCALEer and Bartels, 1992; and MCaLEER, 1992, for further details). Kruskal's theorem states that OLS is efficient for $\left(\delta_{0}, \beta_{2}\right)$ if and only if:

(i) $\Sigma_{\varepsilon} \hat{y}_{1}=\hat{y}_{1} A_{1}, \quad$ for some $A_{1}$,

(ii) $\Sigma_{\varepsilon} \hat{X}_{2}=\hat{X}_{2} A_{2}$, for some $A_{2}$.

The Gauss-Markov theorem is a special case of Kruskal's theorem, and hence is sufficient for OLS to be efficient. In the context of OLS estimation of equation (8), the necessary and sufficient conditions for OLS to be efficient are given as follows.

Proposition 1. OLS in (8) is efficient if and only if conditions (i) and (ii) hold simultaneously.

Proof.

(i) $\quad \Sigma_{\varepsilon} \hat{y}_{1}=\left(\sigma_{2}^{2} I+\beta_{2}^{2} \sigma_{\eta}^{2} P_{W}\right) P_{1} y$

$$
\begin{aligned}
& =P_{1} y\left(\sigma_{2}^{2} I+\beta_{2}^{2} \sigma_{\eta}^{2}\right) I=P_{1} y A_{1}, \quad \text { if } X_{1} \subset W \text {; } \\
& =P_{1} y\left(\sigma_{2}^{2} I\right)=P_{1} y A_{1}, \quad \text { if } X_{1} \perp W \text {. }
\end{aligned}
$$

() 2009 The Authors. Journal compilation (c) 2009 VVS. 
(ii) $\quad \Sigma_{\varepsilon} \hat{X}_{2}=\left(\sigma_{2}^{2} I+\beta_{2}^{2} \sigma_{\eta}^{2} P_{W}\right) P_{W} X_{2}$

$$
\begin{aligned}
& =P_{W} X_{2}\left(\sigma_{2}^{2}+\beta_{2}^{2} \sigma_{\eta}^{2}\right) I \\
& =\hat{X}_{2} A_{2} .
\end{aligned}
$$

The necessary and sufficient conditions (i) and (ii) are satisfied either if $X_{1} \subset W$ or if $X_{1} \perp W$ (see PAGAN, 1984, for the case of generated regressors, and MCALEer and MCKENZIE, 1991, for a simple proof of efficiency of related two-step estimators), so that OLS estimation of the parameters in equation (8) is efficient.

Remark 10 . Of the two necessary and sufficient conditions, it is more likely that $X_{1} \subset W$ will hold as $\hat{y}_{1}=X_{1} \hat{\beta}_{1} \subset W$, whereas orthogonality between $X_{1}$ and $W$ (i.e. $\left.X_{1} \perp W\right)$ is not possible by virtue of $\hat{y}_{1} \subset W$,

Let $X_{3}=\left[\hat{y}_{1}: \hat{X}_{2}\right]$ in equation $(8)$ be a $(T \times 2)$ matrix, and let $\beta_{3}=\left(\delta_{0}, \beta_{2}\right)$ be a $(2 \times 1)$ vector, so that equation $(8)$ can be written as:

$$
y=X_{3} \beta_{3}+\varepsilon \text {. }
$$

Regarding inference, the OLS covariance matrix for equation (12) is given by

$$
\operatorname{var}\left(\hat{\beta}_{3, \text { OLS }}\right)=\left(X_{3}^{\prime} X_{3}\right)^{-1} X_{3}^{\prime} \Sigma_{\varepsilon} X_{3}\left(X_{3}^{\prime} X_{3}\right)^{-1} \text {. }
$$

Substituting for $\Sigma_{\varepsilon}$ from equation (11) in equation (13) gives

$$
\operatorname{var}\left(\hat{\beta}_{3, \mathrm{OLS}}\right)=\sigma_{2}^{2}\left(X_{3}^{\prime} X_{3}\right)^{-1}+\beta_{2}^{2} \sigma_{\eta}^{2}\left(X_{3}^{\prime} X_{3}\right)^{-1} X_{3}^{\prime} P_{W} X_{3}\left(X_{3}^{\prime} X_{3}\right)^{-1} .
$$

REMARK 11. If the incorrect downward-biased OLS standard errors are used, namely from $\sigma_{2}^{2}\left(X_{3}^{\prime} X_{3}\right)^{-1}$, then the $t$-ratios for $\hat{\beta}_{3, \mathrm{OLS}}$ will be biased upward (a similar result was given in Pagan, 1984, for generated regressors; see also OxLey and Mcaleer, 1993).

REMARK 12. The covariance matrix in equation (14) may be estimated consistently using the Newey-West HAC standard errors. In practice, the HAC standard errors may not be accurate in the context of generated regressors, so that equation (14) should be calculated for purposes of testing hypotheses and constructing confidence intervals (see SMith and McaleER, 1994, for further details).

\subsection{A Direct test of expertise on expert opinion}

The analysis presented before relates to generating an REF, and a test of the significance of the REF, in explaining $y$. Expert opinion, as manifested in $X_{2}$, can be tested separately by substituting from equation (3) into equation (1) to give

$$
y=\delta_{0} \hat{y}_{1}+\beta_{2} X_{2}+\left(u_{2}-\beta_{2} \eta\right) .
$$

OLS will be inconsistent in equation (15) as $X_{2}$ is correlated with $\eta$ through equation (3). Therefore, IV should be used whenever expert opinion is used to fore(c) 2009 The Authors. Journal compilation (c) 2009 VVS. 
cast the variable of interest. In empirical practice, OLS rather than IV is typically used, incorrectly, to estimate the parameters in equation (15). Moreover, under a MSE loss function, the forecast of $y$ in equation (15) is not optimal relative to the information set $\left(\hat{y}_{1}, X_{2}\right)$.

The effect of expertise on expert opinion can be tested directly by testing appropriate hypotheses in equation (4), which may be rewritten as:

$$
X_{2}=W \delta+\eta=\delta_{0} \hat{y}_{1}+W_{1} \delta_{1}+\eta, \quad \eta \sim\left(0, \sigma_{\eta}^{2} I\right) .
$$

OLS is efficient for $\delta_{0}$ and $\delta_{1}$ in equation (16), and various null hypotheses, such as

$$
H_{0}: \delta_{0}=\delta_{0}^{*},
$$

can be tested directly. Interesting values of $\delta_{0}^{*}$ would be 0 or 1 , meaning that the expert fully discards the model forecast or fully adopts it, respectively.

Under a MSE loss function, the forecast of $X_{2}$ in equation (16) is optimal relative to the expert's information set, $I_{-1}^{E}$. A direct test of expertise, namely whether the expert adds any additional information to $\hat{y}_{1}$ in formulating expert opinion, $X_{2}$, is given by

$$
H_{0}: \delta_{1}=0 .
$$

If the null hypothesis in equation (17) is not rejected, expertise does not add significantly to $\hat{y}_{1}$ in determining the expert opinion, regardless of the value of $\delta_{0}$.

Remark 13. The auxiliary regression equation used in Blattberg and Hoch (1990), namely to correlate expert opinion and model-based econometric forecasts, can be written as:

$$
X_{2}=\delta_{0} \hat{y}_{1}+v .
$$

In comparison with equation (16), it is clear that OLS applied to equation (18) omits $W_{1}$, which denotes expertise in the information set of the expert. As it is highly likely that $W_{1}$ and $\hat{y}_{1}$ are correlated, OLS will be inconsistent and inferences will be invalid.

For equations (16) and (18) to be equivalent, it follows that

$$
v=W_{1} \delta_{1}+\eta,
$$

in which case expertise cannot be tested in equation (18) as it is not included in the specification. It is also quite likely that $v$ in equation (18) will be serially correlated, especially if the missing $W_{1}$ contains lagged values of variables (see Franses and Legerstee, 2009, for empirical evidence of such serial correlation). Therefore, inferences based on equation (18) will be biased and invalid. Moreover, under a MSE loss function, the forecasts from equation (18) will not be optimal. 


\section{Empirical example}

The estimation, testing and forecasting methods described before are illustrated in this section using data for three experts who provide their expert forecasts, $X_{2}$, after they have been given the model forecasts, $\hat{y}_{1}$. The three experts are employed by a Netherlands-based pharmaceutical company, and are based in the Netherlands, Germany and Sweden. They are responsible for the supply chain management in local offices, and hence need to have accurate forecasts for the monthly sales of various products. The company offers products within seven distinct categories, and each expert is responsible for the products within a single category. Each month, the company's headquarters deliver the one-step-ahead model forecasts, and the experts are permitted to provide different quotes. The company uses an automated programme that creates model-based forecasts, where the forecasting scheme can be based on ARIMA models, exponential smoothing, Holt-Winters techniques, and several other standard forecasting methods. The input variables of the models are lagged sales only. Each month, the programme estimates a range of models, and selects the model with the best in-sample forecasting performance. Hence, parameter estimates are updated each month. The experts are aware of how the company creates their forecasts, so that the model forecasts are contained in their respective information sets.

The sample is from October 2004 to October 2006. The three experts in our sample are responsible for a different number of products, ranging from around 210 for the Dutch and Swedish experts, to around 800 for the German expert. We have data on actual sales, $y$, the model forecast $\hat{y}_{1}$, and the expert forecast $X_{2}$. In expertise, $W_{1}$, we include $y_{t-2}$ (as this is known to the expert at the time when a forecast is made for time $t), X_{2, t-2}-y_{t-2}, \hat{y}_{1, t-2}-y_{t-2}$, and $X_{2, t-1}-\hat{y}_{1, t-1}$.

Table 1 provides a comparison of the model and expert forecasts in terms of median squared prediction error. The mean squared prediction error is contaminated by a few outliers. It is clear that the three experts provide far superior forecasts than the model used by the headquarters. In this sense, the experts seem to know what they are doing.

The results for regression equations (4) and (18), namely the separate effects of the model forecast and expertise on expert opinion, are reported in Table 2. The estimates for equation (18) are biased and inconsistent, and inferences are invalid, because of the omitted variables bias. For all three experts, it would appear that the effect of the model forecast is extremely close to unity in the absence of exper-

Table 1. A comparison of model and expert forecasts.

\begin{tabular}{llr}
\hline & \multicolumn{2}{c}{ Median squared prediction errors } \\
\cline { 2 - 3 } Country-category & \multicolumn{1}{c}{ Model } & Expert \\
\hline Expert 1 & 203,855 & 28,731 \\
Expert 2 & 197,136 & 166,464 \\
Expert 3 & 17,031 & 15,751 \\
\hline
\end{tabular}

(C) 2009 The Authors. Journal compilation (® 2009 VVS. 
Table 2. Testing the effect of expertise on expert opinion (standard errors are in parentheses)

\begin{tabular}{|c|c|c|c|c|c|c|}
\hline \multirow[b]{2}{*}{ Included variables } & \multicolumn{2}{|l|}{ Expert 1} & \multicolumn{2}{|l|}{ Expert 2} & \multicolumn{2}{|l|}{ Expert 3} \\
\hline & (4) & (18) & (4) & (18) & (4) & (18) \\
\hline Intercept & $\begin{array}{c}-22.34 \\
(77.59)\end{array}$ & $\begin{array}{r}95.56 \\
(128.5)\end{array}$ & $\begin{array}{c}-26.43 \\
(59.46)\end{array}$ & $\begin{array}{l}207.7 \\
(90.04)\end{array}$ & $\begin{array}{c}42.08 \\
(39.88)\end{array}$ & $\begin{array}{l}119.3^{*} \\
(49.47)\end{array}$ \\
\hline Model forecast $(t)$ & $\begin{array}{c}0.09 \\
(0.05)\end{array}$ & $\begin{array}{l}0.97^{* *} \\
(0.01)\end{array}$ & $\begin{array}{l}0.24^{* *} \\
(0.03)\end{array}$ & $\begin{array}{l}1.01^{* *} \\
(0.00)\end{array}$ & $\begin{array}{l}0.30^{* *} \\
(0.10)\end{array}$ & $\begin{array}{l}0.96^{* *} \\
(0.01)\end{array}$ \\
\hline Sales $(t-2)$ & $\begin{array}{l}0.91^{* *} \\
(0.05)\end{array}$ & & $\begin{array}{l}0.78^{* *} \\
(0.03)\end{array}$ & & $\begin{array}{l}0.67 * * \\
(0.10)\end{array}$ & \\
\hline Model forecast & $0.19 * *$ & & $0.16^{* *}$ & & $0.47 * *$ & \\
\hline Minus sales $(t-2)$ & $(0.03)$ & & $(0.03)$ & & 0.06 & \\
\hline Expert forecast & $0.59 * *$ & & $0.46^{* *}$ & & $0.26^{* *}$ & \\
\hline Minus sales $(t-2)$ & $(0.05)$ & & $(0.03)$ & & $(0.07)$ & \\
\hline & -0.07 & & $0.17 * *$ & & $0.25^{* *}$ & \\
\hline Model forecast $(t-1)$ & $(0.04)$ & & $(0.03)$ & & $(0.06)$ & \\
\hline$R^{2}$ & 1.00 & 0.98 & 0.99 & 0.98 & 0.98 & 0.95 \\
\hline$F$-test & $145.0 * *$ & & $292.5^{* *}$ & & $76.45^{* *}$ & \\
\hline
\end{tabular}

Notes: The regression model correlates the expert opinion, $X_{2}$, and model forecast, $\hat{y}_{1}$, in

$$
X_{2}=a_{0}+b_{0} \hat{y}_{1}+v
$$

but omits the effect of expertise on expert opinion. Expertise is approximated by two-period lagged sales, the model forecast error two periods lagged, expert forecast error two periods lagged, and expert adjustment of the model forecast one period lagged. ${ }^{*}$ and $* *$ denote significance at the $5 \%$ and $1 \%$ levels, respectively. The $F$-test is a test of the omitted expertise variables. (4) and (18) represent equations (4) and (18) in text.

tise (equation (18)), but decreases considerably when expertise is included (equation (4)). Moreover, the $F$-test of excluding the expertise rejects the null hypothesis for all three experts. In short, expertise matters.

Estimates of the model forecast, $\hat{y}_{1}$, and replicable expert forecast, $\hat{X}_{2}$, in predicting the actual values of $y$ are given in Table 3. OLS is efficient, according to the information sets, but the standard errors need to be corrected using the Newey-West HAC formula. The inferences are not qualitatively affected, whether the incorrect OLS or HAC standard errors are used. For Expert 1, the expert forecast dominates the model forecast, which is not significant, whereas for Experts 2 and 3, both the model and replicable expert forecasts are significant. However, in each of the latter two cases, the REF dominates the expert forecast.

Table 4 reports the estimates of the model forecast, $\hat{y}_{1}$, and expert opinion (or Non-REF), $X_{2}$, in predicting the actual values of $y$. As the expert opinion is correlated with the equation error, OLS is inconsistent and generalized method of moments (GMM) is used to provide consistent estimates. The instrument list uses two-period lagged sales, the model forecast error two periods lagged, expert forecast error two periods lagged, and expert adjustment of the model forecast one period lagged. The results are broadly consistent with the estimates presented in Table 3 . For Experts 1 and 3, GMM has the effect of increasing the influence of the expert opinion in predicting actual sales, whereas for Expert 2 it is the reverse. In summary, both model forecasts and expert opinions are important in predicting sales. 
Table 3. Model and replicable expert forecasts in predicting actual values (standard errors are in parentheses)

\begin{tabular}{lclll}
\hline Estimation method & Intercept & Model forecast $\hat{y}_{1}$ & Expert forecast $X_{2}$ & $R^{2}$ \\
\hline Expert 1 & & & & \\
OLS & 159.92 & -0.05 & $1.03^{* *}$ & 0.98 \\
& $(176.54)$ & $(0.09)$ & $(0.09)$ & \\
HAC & {$[138.05]$} & {$[0.19]$} & {$[0.21]$} & 0.96 \\
Expert 2 & 21.10 & $0.42^{* *}$ & $0.51^{* *}$ & \\
OLS & $(111.7)$ & $(0.06)$ & $(0.05)$ & \\
HAC & {$[122.3]$} & {$[0.16]$} & {$[0.14]$} & 0.99 \\
Expert 3 & & & & \\
OLS & $-82.94^{* *}$ & $0.30^{* *}$ & $0.66^{* *}$ & $(0.05)$ \\
HAC & $(30.78)$ & $(0.05)$ & {$[0.07]$} & \\
\hline
\end{tabular}

Notes: The regression model is:

$$
y=a+b \hat{y}_{1}+c \hat{X}_{2}+e .
$$

** denotes significance at the $5 \%$ levels. The Newey-West HAC standard errors are given in brackets.

OLS, ordinary least squares.

Table 4. Model and expert forecasts in predicting actual values (standard errors are in parentheses)

\begin{tabular}{lcccr}
\hline Estimation method & Intercept & Model forecast $\hat{y}_{1}$ & Expert Forecast $X_{2}$ & $R^{2}$ \\
\hline Expert 1 & & & & \\
OLS & 104.9 & 0.07 & $0.92^{* *}$ & 0.97 \\
& $(177.0)$ & $(0.08)$ & $(0.08)$ & 0.97 \\
GMM & 150.2 & -0.18 & $\left(0.35^{* *}\right.$ & \\
Expert 2 & $(102.0)$ & $(0.28)$ & & $0.966^{* *}$ \\
OLS & 22.92 & $0.37^{* *}$ & $(0.04)$ & 0.96 \\
GMM & $(101.1)$ & $(0.04)$ & 0.26 & 0.96 \\
Expert 3 & 21.41 & $0.68^{* *}$ & $(0.17)$ & \\
OLS & $(95.08)$ & $(0.18)$ & & 0.97 \\
GMM & -9.28 & $0.52^{* *}$ & $\left(0.43^{* *}\right.$ & 0.98 \\
& $(43.63)$ & $(0.06)$ & $0.65^{* *}$ & \\
\hline
\end{tabular}

Notes: The regression model is:

$$
y=\varphi+\delta_{0} \hat{y}_{1}+\beta_{2} X_{2}+e .
$$

The instrument list uses two-period lagged sales, the model forecast error two periods lagged, expert forecast error two periods lagged, and expert adjustment of the model forecast one period lagged. ${ }^{* *}$ denotes significance at the $5 \%$ level. OLS, ordinary least squares; GMM, generalized method of moments.

\section{Conclusion}

Expert opinion is an opinion given by an expert, and hence can have significant value in forecasting key policy variables in economics and finance. Expert forecasts can either be expert opinions, or forecasts based on an econometric model. An expert forecast that is based on an econometric model is replicable, and can be 
defined as an REF, whereas an expert opinion that is not based on an econometric model can be defined as a Non-REF. Both REF and Non-REF may be made available by an expert regarding a policy variable of interest.

In this paper, we developed a model to generate REF, and compared REF with Non-REF. A method was presented to compare REF and Non-REF using efficient estimation methods, and a direct test of expertise on expert opinion was given. Illustrations for forecasting pharmaceutical SKU, where the econometric model is of the ARIMA type, highlighted the ease of implementation of the estimation and testing procedures developed in the paper, and showed the relevance of the new methodology. In particular, the experts were found to possess significant expertise, and expert forecasts were significant in explaining actual sales.

We foresee two areas for further research. The first is to allow the contribution of the expert to change over time, making some of the parameters time-varying. A second issue concerns an investigation into which aspects of an expert make them a good forecaster. Is it experience, or is it moderate behavior (meaning little adjustment, only when it matters)?

\section{Acknowledgement}

The second author wishes to thank the Australian Research Council for financial support, and the Erasmus School of Economics for their gracious hospitality and excellent working environment.

\section{References}

BlattberG, R. C. and S. J. Hoch (1990), Database models and managerial intuition: 50\% model + 50\% manager, Management Science 36, 887-899.

Bunn, D. W. and A. A. SAlo (1996), Adjustment of forecasts with model consistent expectations, International Journal of Forecasting 12, 163-170.

Fiebig, D. G., M. Mcaleer and R. Bartels (1992), Properties of ordinary least squares estimators in regression models with non-spherical disturbances, Journal of Econometrics 54, 321-334.

Franses, P. H. (2008), Merging models and experts, International Journal of Forecasting 24, 31-33.

Franses, P. H. and R. Legerstee (2009), Properties of expert adjustments on model-based SKU-level forecasts, International Journal of Forecasting 25, 35-47.

Franses, P. H., H. Kranendonk and D. Lanser (2007), On the optimality of expert-adjusted forecasts, CPB Discussion Paper 92, Netherlands Bureau for Policy Analysis, The Hague.

Goodwin, P. (2000), Improving the voluntary integration of statistical forecasts, International Journal of Forecasting 16, 85-99.

Hoti, S., M. Mcaleer and D. Slottue (2006), Intellectual property litigation activity in the USA, Journal of Economic Surveys 20, 715-729.

MCALEER, M. (1992), Efficient estimation: the Rao-Zyskind condition, Kruskal's theorem and ordinary least squares, Economic Record 68, 65-72. 
Mcaleer, M. (1995), The significance of testing empirical non-nested models, Journal of Econometrics 67, 149-171.

Mcaleer, M. and C. McKenZie (1991), When are two step estimators efficient?, Econometric Reviews 10, 235-252.

OxLey, L. and M. McaleeR (1993), Econometric issues in macroeconomic models with generated regressors, Journal of Economic Surveys 7, 1-40.

PaGAN, A. R. (1984), Econometric issues in the analysis of regressions with generated regressors, International Economic Review 25, 221-247.

Patton, A. J. and A. Timmermann (2007a), Properties of optimal forecasts under asymmetric loss and nonlinearity, Journal of Econometrics 140, 884-918.

Patton, A. J. and A. Timmermann (2007b), Testing forecast optimality under unknown loss, Journal of the American Statistical Association 102, 1172-1184.

Romer, C. D. and D. H. Romer (2008), The FOMC versus the staff: where can monetary policymakers add value?, American Economic Review: Papers \& Proceedings 98, 230-235.

Smith, J. and M. Mcaleer (1994), Newey-West covariance matrix estimates for models with generated regressors, Applied Economics 26, 635-640.

Received: March 2009. Revised: March 2009. 